\title{
Création de ressources lexicographiques Français-Slovène d'aide à la traduction spécialisée
}

\author{
Sonia Vaupot, Département de traduction, Faculté des Lettres, \\ Université de Ljubljana, Slovénie \\ (sonia.vaupot@ff.uni-lj.si)
}

\begin{abstract}
Résumé: Afin de pallier le manque de ressources lexicographiques pour la paire de langues français-slovène dans le domaine de la traduction spécialisée, un projet bilatéral a été mis en place dans le but de développer un dictionnaire en ligne destiné à être utilisé comme un outil lexicographique et une aide à la rédaction de textes scientifiques. Cet article présente une méthode qui permet aux étudiants de participer activement au processus de création d'une base de données en ligne en vue du stockage de données terminologiques et phraséologiques. Nous présentons tout d'abord la situation lexicographique en Slovénie, notamment pour la paire de langues françaisslovène, puis les objectifs du projet ainsi que la méthode d'enseignement visant la création de corpus comparables et de ressources lexicographiques compilées par les étudiants. Enfin, nous terminons par une synthèse des résultats obtenus. Cette méthode, utilisée depuis 2018 dans le cadre d'un cours de Master, donne d'excellents résultats du point de vue pratique et pédagogique.
\end{abstract}

Mots-clés: DICTIONNAIRE BILINGUE, DICTIONNAIRE MULTILINGUE, FORMATION EN TRADUCTION SPÉCIALISÉE, CORPUS SPÉCIALISÉS, TERMINOLOGIE, PHRASÉOLOGIE, COLLOCATIONS, TRADUCTION, ÉQUIVALENCE, DIDACTIQUE

\footnotetext{
Abstract: Creating a Slovene-French LSP Dictionary for translation purposes. In order to compensate for the lack of lexicographical resources for the French-Slovenian language pair in the field of specialized translation, a bilateral project has been set up with the aim of developing an online dictionary to be used as a lexicographical tool and as an aid for writing scientific texts. This article presents a method that allows students to actively participate in the process of creating an online database for storing information on specialized terminology and phraseology. We first present the lexicographical situation in Slovenia, especially for the FrenchSlovenian language pair, and then the objectives of the project as well as the teaching method aimed at creating comparable corpora and lexicographical resources compiled by the students. Finally, we conclude with a synthesis of the results obtained. This method, which has been used since 2018 in a Masters course, provides excellent results from a practical and pedagogical point of view.

Keywords: BILINGUAL DICTIONARY, MULTILINGUAL DICTIONARY, SPECIALISED TRANSLATION TEACHING, SPECIALISED CORPORA, TERMINOLOGY, PHRASEOLOGY, COLLOCATIONS, TRANSLATION, EQUIVALENCE, DIDACTICS
} 


\section{Introduction}

La Slovénie possède une certaine tradition lexicographique qui s'est mise en place au fur et à mesure des contacts avec la langue allemande dans le contexte culturel de l'Europe centrale. Pays de plus de deux millions d'habitants, ce pays a été sous la domination du Saint Empire romain germanique et des Habsbourg d'Autriche, avant de faire partie de la Yougoslavie de 1918 à 1991, date de son indépendance.

Créée en 1938, l'Académie slovène des sciences et des arts (en slovène, Slovenska akademija znanosti in umetnosti ou SAZU) comprend l'Institut de la langue slovène Fran Ramovš, fondé en 1945, dont l'une des missions concerne la création de bases de données lexicographiques. Cet Institut a participé à plusieurs projets nationaux et internationaux, dont l'Atlas linguistique slave général (OLA), l'Atlas Linguarum Europae (ALE), la Fédération internationale des banques de terminologie (IFTB), l'Encyclopédie onomastique slovène (EOS), etc. Depuis la proclamation de l'indépendance en 1991, les Slovènes ont continué à développer un intérêt croissant pour leur langue. En 2004, l'Institut d'études appliquées Trojina a été fondé à Ljubljana dans le but de promouvoir la linguistique contemporaine. Orienté vers la recherche et le développement de la langue slovène, l'Institut a collaboré à plusieurs reprises avec l'Université de Ljubljana sur des projets lexicographiques. De nombreux projets (dictionnaires monolingue, bilingue, phraséologique, etc.) ont ainsi vu le jour. Parallèlement, des travaux de recherche (Vrbinc et al. 2018: 363) ont été menés sur la lexicographie slovène en général (Snoj 2004; Žagar Karer 2011; Ledinek et al. 2015; Srebnik 2015; Gantar 2015; Gorjanc et al. 2017). Ces travaux sont influencés, entre autres, par les théories lexicographiques: britannique (Sue Atkins, Patrick Hanks, RRK Hartmann, Adam Kilgarriff, Michael Rundell et John Sinclair); francophone (Sylviane Granger, Gilles-Maurice de Schryver); tchèque (František Čermák, Ladislav Zgusta); allemande (Herbert Ernst Wiegand, Ute Römer); américaine (Dwight Bolinger, Don McCreary, Erin McKean et Ben Zimmer) et russe (Juri Apresjan). Quant à la terminographie moderne, elle subit l'influence de l'école de terminologie de Vienne (ibid.: 364-365).

Toutefois, depuis l'adhésion de la Slovénie à l'Union européenne, un besoin conséquent en ressources lexicales et terminologiques bilingues et plurilingues apparait notamment dans le domaine de la traduction. Malgré les nombreuses ressources disponibles, on constate une pénurie de ressources lexicales générales et spécialisées, plus particulièrement pour la paire de langues français-slovène ou inversement. Actuellement, les dictionnaires suivants sont disponibles, en version papier ou électronique: le dictionnaire français-slovène (Grad 2004, la version papier datant de 1971), le dictionnaire slovène-français (Jesenik et Dembskij 2008; la version papier datant de 1990) ainsi qu'une édition de poche (Perko 2006) et le dictionnaire en ligne Pons. Il existe peu de dictionnaires spécialisés, mentionnons le dictionnaire slovène-français européen (Perko 2007) et le dictionnaire terminologique slovène-anglais-français (version pdf, 2007) reprenant la terminologie de la Présidence slovène et accessible sur 
le site du Secrétariat général du gouvernement de la République slovène, ainsi que les bases multilingues Glosbe et Linguee. Il est aussi possible de consulter en ligne les bases de données lexicales multilingues Evrokorpus, Evroterm ou IATE qui se réfèrent essentiellement aux corpus communautaires.

Par ailleurs, face à la taille du marché de la traduction en Slovénie, il est nécessaire de préciser que les jeunes traducteurs peuvent difficilement se spécialiser dans un seul domaine. Ils sont en grande majorité polyvalents et amenés à traduire, dans des domaines très variés, aussi bien dans la langue maternelle que dans une langue étrangère. Les différents bureaux et services de traduction privés ou officiels (bureau de traduction du gouvernement, différents ministères, etc.) exigent parfois de savoir traduire dans une voire plusieurs langues étrangères. La majorité des traductions dans la langue étrangère sont ensuite relues par un réviseur professionnel. Il est donc indispensable de former les étudiants, futurs traducteurs, à traduire différents types de textes vers la langue maternelle et vers la langue étrangère. Le Département de traduction de la Faculté des Lettres de l'Université du Ljubljana (Slovénie) accueille ainsi chaque année des étudiants qui ont pour langues obligatoires, le slovène (langue maternelle $A$ ) et l'anglais (langue étrangère $B$ ). Les étudiants choisissent également une autre langue B: l'allemand, le français ou l'italien. Au cours de leur cursus, ils sont donc amenés à traduire vers leur langue $\mathrm{A}$ et leurs deux langues B. Après leurs études de Master, une fois sur le marché du travail, les jeunes traducteurs doivent être capables non seulement de traduire tout type de textes, mais aussi d'assurer la gestion terminologique et de pouvoir utiliser divers outils de traduction (Trados, par exemple).

Cet article expose, d'une part, les résultats de deux enquêtes menées auprès d'étudiants inscrits en Master de traduction, l'objectif étant d'identifier la situation lexicographique actuelle pour la paire de langues français-slovène. D'autre part, le marché de la traduction intégrant celui de la création de ressources linguistiques (monolingues, bilingues ou multilingues), nous présentons le projet bilatéral Proteus mis en place entre l'UFR EILA de l'Université de Paris (anciennement Paris 7-Diderot) et le département de traduction de l'Université de Ljubljana. Ce projet porte sur la conception de ressources lexicographiques français-slovènes dans le dictionnaire en ligne ARTES. Nous présentons une méthode active et créative visant à familiariser l'étudiant avec la terminologie et la phraséologie tout en créant un dictionnaire spécialisé bilingue en ligne. Les ressources dans la base sont en effet compilées par les étudiants. Nous présentons les objectifs du projet ainsi que la méthode d'enseignement visant la création des corpus comparables et des ressources lexicographiques en ligne. Enfin, nous terminons par une synthèse des résultats obtenus.

\section{Enquêtes exploratoires}

Face au peu de ressources lexicographiques à disposition pour le couple de langue français-slovène et inversement, deux enquêtes ont été menées durant 
deux années consécutives (de 2018 à 2020). Nous présentons ici les résultats d'une première enquête ayant pour objectif de vérifier l'état actuel du dictionnaire français-slovène (1971), puis une autre enquête qui visait à identifier la méthode de travail des étudiants pour traduire un texte spécialisé sans dictionnaire terminologique à leur disposition.

\subsection{Faiblesses du dictionnaire bilingue}

L'importance des locutions dans l'apprentissage d'une langue étrangère n'est plus à prouver (Galisson 1983 et 1991; Tréville 2000; Boers, Demecheleer et Eyckemans 2004; Detry 2008; Pavičić Takač 2008, Cavalla 2009, Szczepaniak et Lew 2011, Simonnet et Tutin 2019, etc.). Nous savons également à quel point la traduction des locutions peut poser des difficultés. Nous avons ainsi choisi de vérifier les traductions slovènes des locutions verbales françaises (LVF), proposées par le dictionnaire français-slovène dont la version papier date de 1971 et la version électronique de 2004.

L'un des premiers réflexes étant de faire une recherche dans le dictionnaire avant de traduire une expression figée inconnue, nous avons proposé un exercice aux étudiants inscrits en Licence 2 et Licence 3 qui consistait à traduire vers leur langue maternelle $A$ (le slovène) 115 locutions verbales françaises avant même de consulter le dictionnaire bilingue. Cette approche visait à vérifier le traitement et la traduction des locutions verbales dans le dictionnaire en ligne slovène-français (Grad 2004). En s'aidant de la définition ou l'explication proposée par le dictionnaire unilingue français de leur choix, les apprenants ont proposé leurs propres traductions slovènes des LVF. Les résultats ont ensuite été recueillis dans un fichier Excel, comportant les LVF, leur définition ou l'explication tirée d'un dictionnaire français, la traduction de l'étudiant et la traduction proposée par le dictionnaire bilingue. En comparant les traductions proposées par le dictionnaire slovène et celles des apprenants, notre objectif visait non seulement à explorer les stratégies de traduction des LVF, mais aussi à vérifier les traductions proposées par le dictionnaire bilingue dont la première version, datant de 1971, a été éditée à 9 reprises. Les résultats sont présentés dans le Tableau 1.

Cette approche nous a permis de constater les faiblesses du dictionnaire bilingue: sur les 115 LVF, 48 LVF sont absentes du dictionnaire (soit un peu moins de la moitié). Parmi les LVF qui apparaissent dans le dictionnaire, on compte 38 LVF traduites en slovène par un simple verbe; 13 LVF sont explicitées; 10 sont traduites par une expression verbale non figée et 6 LVF sont traduites par une expression idiomatique figée. Si nous comparons ces résultats avec les traductions des étudiants, nous remarquons que la majorité des LVF ont été traduites, et non explicitées, par une expression slovène équivalente. Les résultats de cet exercice montrent clairement que la proportion des LVF est non seulement faible dans le dictionnaire bilingue, mais la majorité des expressions sont expliquées au lieu d'être traduites. Nous en concluons que le diction- 
naire bilingue s'intéresse aux informations linguistiques et sémantiques, mais ne tient pas suffisamment compte du côté idiomatique de la langue. En ce qui concerne les LVF, la micro-structure est réduite, limitée à une partie explicative et ne répond pas vraiment aux besoins de traduction.

Tableau 1: Les LVF dans le dictionnaire bilingue français-slovène (Grad 2004)

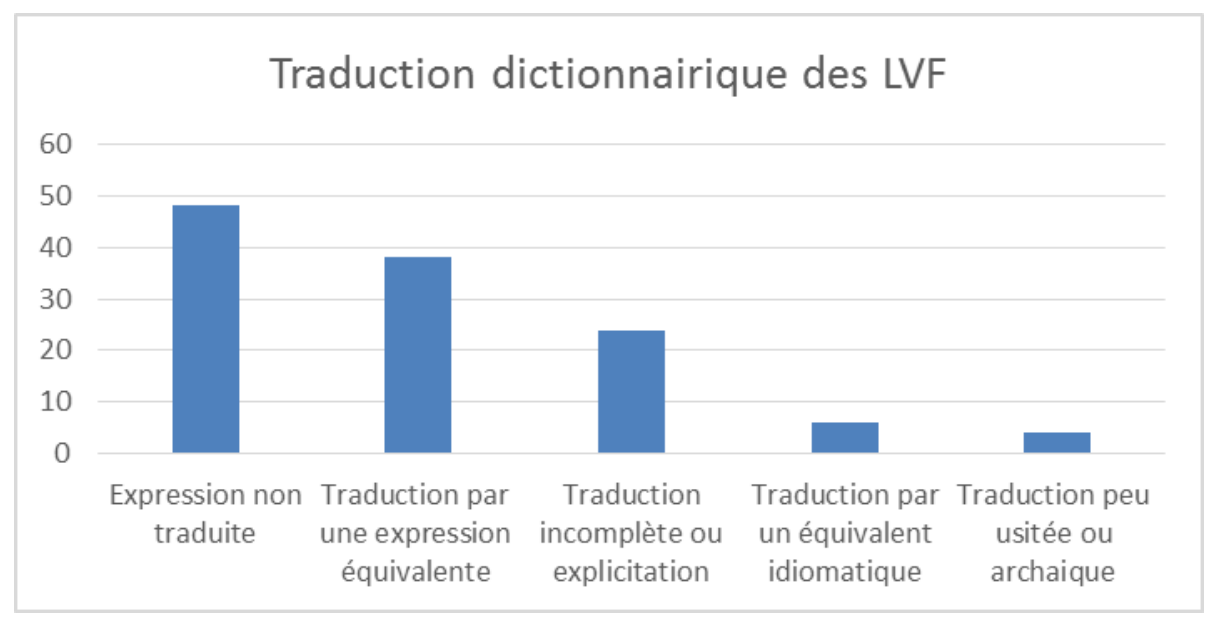

\subsection{Manque de ressources terminologiques}

Une deuxième approche informatisée a été effectuée à l'aide de la méthode d'enregistrement in vivo Translog afin de collecter les données pendant l'opération traduisante (corrections, révisions, pauses, etc.) et notamment afin de vérifier la fréquence des outils en ligne utilisés par les apprenants (voir aussi Jakobsen 1999, 2000). Les étudiants, inscrits en première et deuxième années de Master, ont été priés de traduire à partir du slovène vers leur seconde langue étrangère $B$ (le français) un court texte comprenant une terminologie spécialisée relative à la région du Karst en Slovénie (Mavrič Bordon 2019). Les données recueillies par le logiciel Translog ont permis de constater que les apprenants ont utilisé, par ordre de préférence: le dictionnaire multilingue Glosbe, le dictionnaire électronique slovène-français (Jesenik et Dembskij 2008), le dictionnaire Pons et la base multilingue Linguee. Certains apprenants sont passés par une langue intermédiaire, présentement l'anglais, et ont utilisé les dictionnaires multilingues Word reference et Linguee ainsi que le portail multilingue Termania. Ils ont également vérifié certains termes français à l'aide du Petit Robert ou du Petit Larousse en ligne. Enfin, le dictionnaire multilingue Evroterm et le dictionnaire anglais-français Larousse n'ont été utilisés qu'à une seule reprise. Le Tableau 2 présente les dictionnaires utilisés par les étudiants selon leur fréquence: 
Tableau 2: Outils en ligne selon leur fréquence d'utilisation

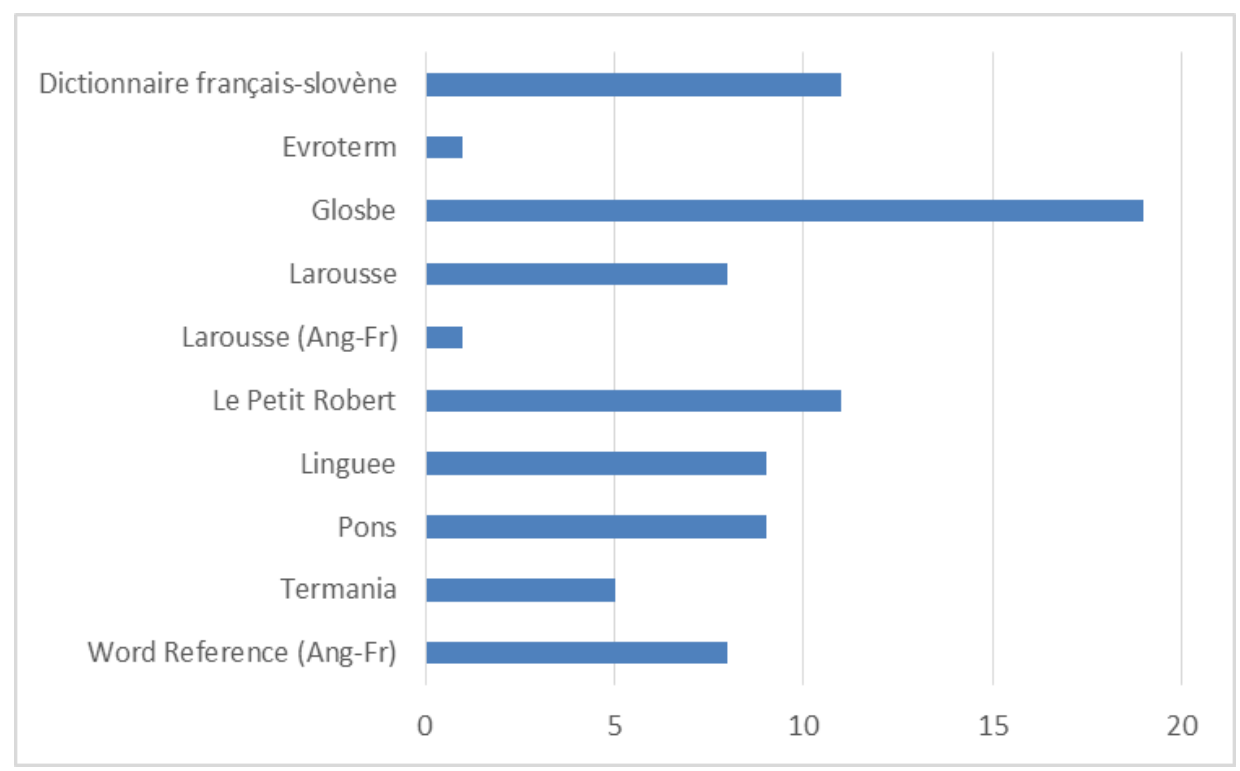

Aucun de ces dictionnaires n'étant spécialisé dans la terminologie du Karst, nous pouvons confirmer la tendance des étudiants à utiliser des dictionnaires généraux en ligne et à récolter ensuite les termes spécialisés par l'intermédiaire d'un moteur de recherche (Google, par exemple). En plus des dictionnaires en ligne, les apprenants ont tous consulté Wikipédia, trois apprenants ont parcouru chacun un article scientifique différent et deux apprenants ont consulté la traduction française d'un texte touristique sur le sujet. Ceci leur a permis de trouver une partie de la terminologie requise. Nous remarquons également que les étudiants n'ont pratiquement pas eu recours à un corpus pour traduire leur texte.

Fort de ces constats, la nécessité d'élaborer une base de données lexicographique s'est imposée afin de mettre en place un outil de traduction plus spécialisé pour la combinaison linguistique slovène et française.

\section{Conception du projet lexicographique}

Afin de pallier le manque de ressources pour le couple de langue françaisslovène, notamment dans le domaine de la traduction spécialisée, un projet bilatéral Proteus a été conduit en 2017 entre le département de traduction de la Faculté des lettres de l'Université de Ljubljana (Slovénie) et l'UFR EILA de l'Université de Paris (autrefois Université de Paris 7-Diderot). Ce projet a pour objectif principal l'élaboration d'un dictionnaire bilingue spécialisé qui serait à 
la fois un outil pour les traducteurs et une aide à la rédaction professionnelle (Pecman 2007, 2012; Pecman et Kübler 2011; Pecman et Gledhill 2018; Kübler 2003, 2011; Kübler et Pecman 2012). Dans un premier temps, cela consiste à développer des ressources terminologiques et phraséologiques français-slovènes (et inversement) d'aide à la traduction spécialisée au moyen du dictionnaire en ligne ARTES. L'équipe de l'UFR EILA de l'Université de Paris a aimablement autorisé et permis l'accès à la base ARTES. En plus de l'outil informatique, elle a également apporté les méthodes et son expérience puisqu'elle mène ce projet depuis plusieurs années dans les domaines de la terminologie et phraséologie spécialisées, entre autres, pour la combinaison linguistique français-anglais et inversement. La base en ligne est également conçue pour prévoir des collaborations extérieures multilingues et est adaptée à une cinquantaine de langues. L'accès à la base est assuré par l'équipe de recherche CLILLAC.

Les ressources dans la base ARTES sont en libre accès, sous forme de fiches terminologiques ou d'entrées de dictionnaires, par le biais de son interface de consultation (https://artes.app.univ-paris-diderot.fr/artes-symfony/ web/app.php). L'interrogation peut porter sur les termes depuis l'onglet «Dictionnaires des termes» ou sur les structures lexico-grammaticales transversales aux domaines, appelées également 'collocations génériques', depuis l'onglet «Dictionnaire des expressions». La fonction «Recherches multicritères», accessible depuis l'interface de consultation (https://www.eila.univ-paris-diderot.fr/ recherche/artes/index) permet également d'interroger ces ressources au moyen une série de requêtes visant à définir les critères de recherche.

Tableau 3: L'interface de consultation de l'outil ARTES

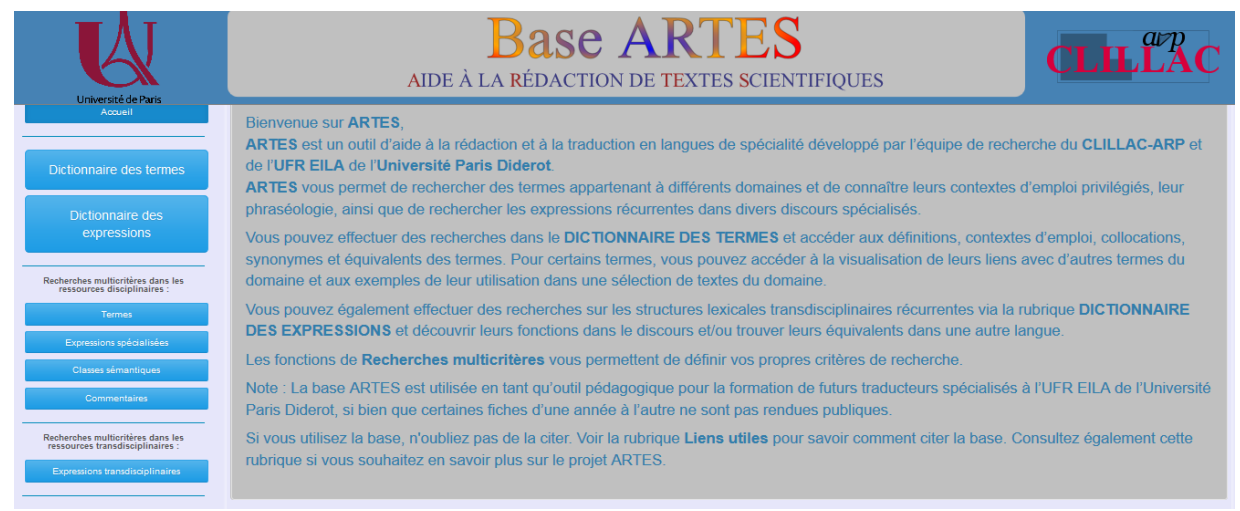

Dans le cadre du projet bilatéral, la compilation de données dans la base ARTES a été conçue pour répondre aux besoins lexicographiques et pédagogiques spécifiques du département de traduction. Le projet a nécessité la mise en place d'une plateforme pour partager les spécifications et les res- 
sources, ainsi que d'un calendrier pour prévoir un échange efficace entre les divers participants. La formation, le soutien à distance et le suivi de la base de données lexicographiques sont assurés par l'équipe française. Des profils d'utilisateurs sont ainsi créés chaque année afin de permettre aux étudiants d'alimenter la base. La base ARTES étant conçue pour permettre aux apprenants de participer au projet, la création des fiches terminologiques et phraséologiques est ainsi confiée, chaque année, aux étudiants inscrits en Master de traduction sous la direction de l'enseignante slovène responsable du projet.

En plus de l'intérêt professionnel, les plus-values didactique et pédagogique du dictionnaire ne sont pas à négliger. Les étudiants sont régulièrement initiés à la recherche d'informations, à la linguistique de corpus et à la création de ressources lexicales. Ils développent des compétences et acquièrent des connaissances souvent indispensables en traduction spécialisée. Le résultat final est présenté sous la forme d'un dossier que les étudiants doivent remettre à l'issue du cours. Les enseignants peuvent ainsi suivre les travaux en cours et évaluer les ressources compilées par les apprenants.

L'objectif final du projet étant d'élaborer une base de données lexicographique, la méthodologie utilisée pour assurer la qualité des données collectées par les apprenants est répartie en trois étapes clés que nous présentons par la suite: (1) la création des corpus comparables, l'analyse et l'extraction des termes ou collocations; (2) la saisie des termes et/ou des collocations dans le dictionnaire en ligne ARTES; (3) la remise des dossiers: des instructions détaillées sont données en début de semestre, environ 3 mois avant la date finale de remise des dossiers.

\section{Recherches et constitution des corpus}

L'intérêt du recours au corpus, notamment pour la traduction spécialisée, a été souligné à maintes reprises et n'est plus à démontrer (Baker 1993; Olohan 2004; Morin et Daille 2006, 2012; Kübler 2011). Rappelons simplement les propos de L'Homme (2004: 119):

La recherche terminographique repose principalement sur le contenu de textes de spécialité. La collecte d'une documentation représentative du domaine dont on souhaite décrire la terminologie et son exploitation constitue les premières étapes d'une recherche en bonne et due forme. Il est désormais possible de réunir une documentation abondante en format électronique dans des délais raisonnables.

En plus des dictionnaires monolingues ou bilingues et des banques terminologiques, les traducteurs peuvent ainsi extraire de nombreuses données provenant de corpus. Il apparait cependant que les corpus sont rarement utilisés par les traducteurs ou futurs traducteurs (Durán-Muñoz 2010) dans leur pratique, notamment les corpus en langue cible. Pourtant, la recherche sur corpus offre l'avantage d'une approche textuelle. En outre, ceux qui travaillent sur la langue 
de spécialité ne peuvent pas toujours procéder par introspection et sont donc dépendants d'une documentation (Meyer et Mackintosh 1996: 265):

Introspection plays a fundamentally different role in terminography than in lexicography, and this has important implications for corpus terminography. Lexicographers, on the one hand, rely heavily on their own linguistic intuition: they are, after all, producers of the type of language they study. Hence, texts and outside informants are often seen as a means of corroborating personal intuition. Terminographers, on the other hand, are not natural producers of the type of language they study. Faced with a terminological problem, they cannot ask questions such as 'what does X mean to me?', 'would I use X in this way?', but rather 'what does $X$ mean to domain experts?', or 'would domain experts use $X$ in this way?'.

Afin d'élaborer une base de données terminologique et phraséologique dans un domaine de spécialité, le recours au corpus semble donc se justifier. Parallèlement à la conception des corpus, un intervenant extérieur, professeur universitaire et spécialiste des domaines de la diplomatie et des relations internationales, est invité chaque année à donner 15 heures de cours aux étudiants de première année de Master en guise d'introduction à un domaine de spécialité (voir Udovič 2018). Les larges connaissances acquises dans les domaines de la diplomatie et des relations internationales permettent aux étudiants de choisir un domaine de spécialité en connaissance de cause avant de constituer leurs corpus, puis de sélectionner et de traiter correctement les termes et les informations pertinentes à ajouter à la base ARTES. En deuxième année de Master, les étudiants puisent la terminologie dans le domaine des sciences de la vie.

Les corpus étant des aides à l'élaboration de dictionnaires bilingues (Bowker et Pearson 2002), ils sont constitués de textes authentiques. Des instructions détaillées sont fournies aux étudiants concernant la création et l'exploitation du corpus. Pour créer leur propre base de données, les apprenants collectent les ressources à partir de corpus textuels. Ils les versent ensuite pour constituer les données à saisir. Ils construisent ainsi deux corpus comparables: français et slovène. Le corpus comparable est composé d'ensembles de textes, dans des langues différentes, «qui ne sont pas des traductions mutuelles» (Bowker et Pearson: 95; voir aussi L'Homme 2004: 133-134), la comparabilité impliquant un nombre de traits communs entre chaque partie du corpus et le seul trait différent étant la langue de chaque partie. Cette définition impose donc peu de contraintes sur les documents constituant le corpus.

La première année du projet, les étudiants ont commencé à moissonner le web pour en extraire les documents, puis à les convertir en texte brut à l'aide du logiciel d'analyse de corpus Antconc. Mais cette approche s'est avérée longue et fastidieuse. La recherche des corpus s'effectue dorénavant à l'aide du logiciel concordancier Sketch Engine qui permet de regrouper facilement de nombreux documents, son utilisation ne nécessitant pas la conversion du document en texte brut. En outre, cet outil est accompagné d'un langage de requêtes puissant, il fournit rapidement des ressources authentiques et permet de régler 
l'affichage des données autour du terme ou de la collocation recherchés. Il s'appuie aussi sur des tableaux regroupant les principaux termes ou cooccurrents classés en fonction de certaines relations (objet, sujet, fréquences, etc.). Les étudiants opèrent donc un tri parmi les ressources proposées par Sketch Engine, et cochent les documents susceptibles d'être intégrés au corpus. Puis, une approche partant des mots clés proposés par le logiciel concordancier est considérée. La recherche se poursuit en fonction des réponses liées à la fréquence d'apparition et/ou à la difficulté, explicite ou implicite, à traduire le terme ou la collocation.

En final, nous notons que les corpus des étudiants (Master 1) portent sur des domaines très variés comme les discours diplomatiques ou politiques, la diplomatie juridique, culturelle, humanitaire ou économique, la politique étrangère, le Brexit, les droits de l'homme, l'impérialisme. etc. Ils sont variables et comptent, en fonction de la langue et du domaine, environ 250000 à 600000 mots chacun.

\section{5. Élaboration du dictionnaire français-slovène en ligne}

La base en ligne ARTES constitue un outil pédagogique destiné à la formation en phraséologie et en terminologie, en plus d'être une ressource lexicographique accueillant de nombreuses informations utiles pour la traduction spécialisée et la rédaction professionnelle. Il est donc indispensable de former les apprenants à l'utilisation de la base qui offre de nombreuses possibilités. L'étudiant peut y construire un simple glossaire ou bien saisir des listes terminologiques ou phraséologiques dans un domaine spécialisé. L'initiation s'effectue en deux temps: d'abord une introduction à la base est effectuée en première année de Master (soit 15h de travaux pratiques sur la base ARTES). En plus de la création des corpus, cela consiste notamment à collecter, saisir et traduire des collocations spécifiques ou génériques. Puis, une application plus poussée est proposée en deuxième année de Master (dans le cadre de 15h de travaux pratiques) où les étudiants produisent des fiches terminologiques dans un domaine de spécialité de leur choix, ces fiches comprenant également la saisie de collocations spécialisées. Ainsi, après la création des corpus, les étudiants recherchent, compilent et traduisent les fiches terminologiques et phraséologiques qu'ils versent dans la base. La conception du dictionnaire débute par la saisie des collocations dans l'onglet «Phraséologie discursive» (Tableau 4). Cela permet une première approche de la base et facilite la saisie ultérieure, plus complexe, de la terminologie dans l'onglet «Terminologie en contexte» (Tableau 6).

\subsection{Création de la base phraséologique discursive}

Des instructions détaillées sont données, au début du semestre, aux étudiants inscrits en première année de Master pour leur apprendre à compiler leurs 
ressources, puis à extraire et saisir les collocations qui figureront dans le dictionnaire. L'étudiant doit ainsi réaliser un projet phraséologique à partir d'un domaine relatif à la diplomatie ou aux relations internationales. Après avoir constitué un corpus comparable, cela consiste à verser dans la base ARTES au moins 5 collocations génériques dans chaque langue source et 5 collocations traduites dans chaque langue cible, donc 10 collocations au total pour les deux langues sources ainsi que leurs traductions. Un problème de définition s'est rapidement posé quant à la nature des collocations, les étudiants peinant à distinguer les collocations spécifiques des collocations génériques ou transdisciplinaires. Plusieurs études ont en effet été menées sur la problématique de la traduction des collocations à partir d'un corpus spécialisé (Kübler 2003, Pecman 2007, Volanschi 2007) qui ont conduit à la séparation des collocations spécifiques (associées à la terminologie) et des collocations génériques (associées au discours).

L'onglet «Phraséologie discursive» de la base ARTES (Tableau 4) étant conçu pour enregistrer les collocations génériques qui apparaissent fréquemment dans le discours, l'étape suivante repose sur l'extraction des collocations les plus fréquentes. L'extraction des collocations consiste à compter le nombre de fois où la base et son collocatif apparaissent dans le corpus. Les apprenants effectuent ensuite un étiquetage morpho-syntaxique afin d'identifier le rôle syntaxique à partir de la morphologie de chaque terme, et un filtrage afin d'éliminer les expressions de faibles fréquences, jugées peu informatives ou ne démontrant pas de réel problème de traduction.

Tableau 4: La phraséologie discursive dans la base ARTES

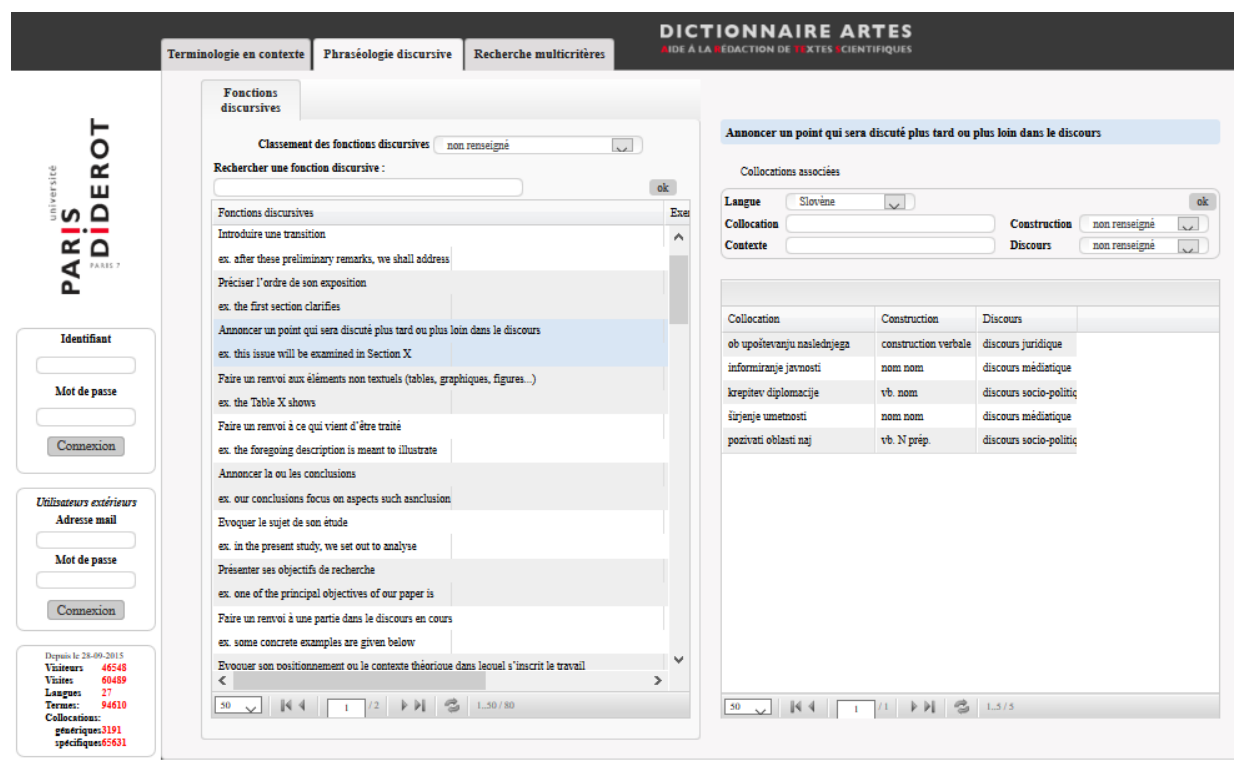


Après avoir été saisie dans la base, chaque collocation est présentée dans un dossier par une fiche qui comprend: la collocation dans la langue source et sa traduction en contexte dans la langue cible, le nombre d'occurrences dans le corpus source et dans Google, la fréquence de la collocation dans la langue cible, les structures lexico-grammaticales et les fonctions discursives. Un exemple de fiche est présenté dans le Tableau 5.

Tableau 5: Exemple de fiche comportant la collocation et sa traduction

\begin{tabular}{|l|l|l|}
\hline & Langue source (français) & Langue cible (slovène) \\
\hline Collocation & permettre de faire vivre & zagotoviti preživetje \\
\hline Fréquence/vos textes/Google & $10 / 15.500 .000$ & 2030 \\
\hline Construction grammaticale & $\mathrm{V}+$ prép + groupe verbal & $\mathrm{V}+\mathrm{N}$ \\
\hline Portée (Artes) & Discuter un concept ou notion & Discuter un concept ou notion \\
\hline
\end{tabular}

Le dossier est à remettre à la fin du semestre. Il est composé d'une introduction, des dix fiches correspondant aux collocations génériques intégrées dans la base et d'une conclusion. Dans l'introduction, l'étudiant présente le domaine, justifient l'intérêt de son choix et la méthode de travail sur corpus. Chaque fiche est suivie d'un commentaire qui porte sur le choix et la traduction de la collocation. L'étudiant tire ensuite des conclusions sur le déroulement des tâches qu'il a accomplies.

\subsection{Création de fiches terminologiques}

L'objectif suivant consiste à créer des fiches terminologiques qui sont versées par les étudiants inscrits en deuxième année de Master dans le dictionnaire en ligne ARTES. La procédure de création des ressources consiste à ajouter manuellement, en tenant compte du contexte, les termes spécialisés et les collocations, puis à identifier leurs équivalents. La saisie du contexte est essentielle pour déterminer le sens et l'usage des unités terminologiques (première étape) qui forment les entrées principales de la base, tandis que les collocations spécifiques (deuxième étape) sont considérées comme des entrées secondaires (Tableau 6). Elles fournissent néanmoins des informations utiles sur les diverses combinaisons de termes dans les situations de communication, notamment dans le domaine des langues de spécialité. 
Tableau 6: Terminologie en contexte dans la base ARTES

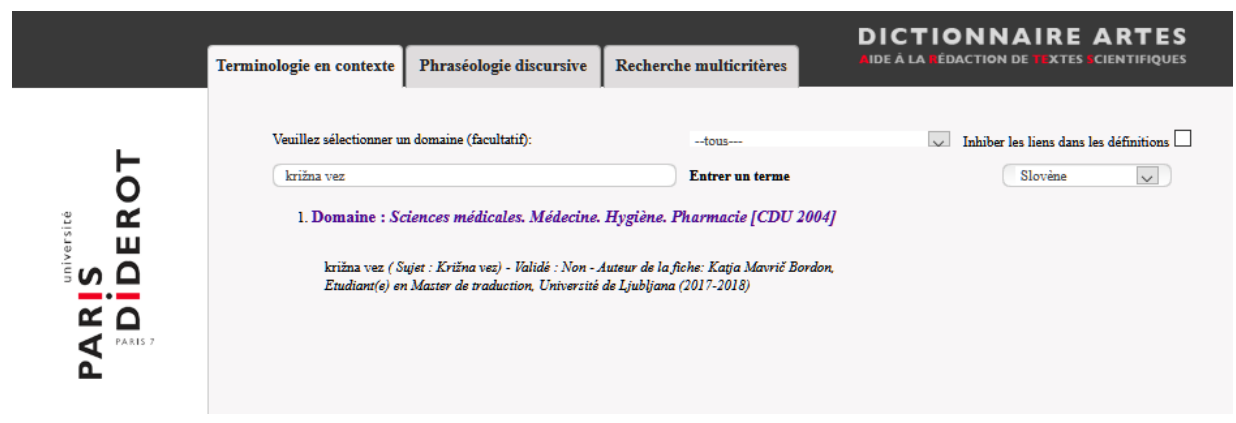

Conformément aux études sur le sujet (L'Homme 2004: 120-123), en établissant leurs corpus, les apprenants sont amenés à collecter et caractériser un ensemble de textes spécialisés jugés pertinents pour l'application visée, à savoir: extraire une liste de termes (simples ou complexes) du domaine de spécialité; repérer les collocations relatives à ces termes et les contextes contenant des éléments définitoires ou des indications sémantiques; repérer les relations sémantiques; vérifier plusieurs équivalents d'un terme; repérer les éléments définitoires, les variantes terminologiques, les indices de relation taxinomique, les synonymes, les co-hyponymes ou des antonymes, les indices de relation méronymique, les termes et leurs cooccurrents.

Par conséquent, l'étudiant doit fournir au moins 10 fiches terminologiques complètes (dites fiches longues) dans chacune des deux langues abordées pour les termes portant sur un domaine spécialisé des sciences de la vie. Le choix du candidat terme est conditionné par le fait que ce terme peut être recherché dans un dictionnaire ou un glossaire pour plusieurs raisons: soit on n'en connait pas le sens ou on hésite sur l'équivalent, soit il est difficile de prouver que la traduction est attestée ou encore que la phraséologie utilisée est correcte. Les apprenants n'ayant que peu d'expérience dans la construction de fiche terminologique, l'une des grandes difficultés réside dans le choix du terme spécialisé. Nous constatons en effet la tendance chez les apprenants à saisir un terme spécialisé qui est similaire dans les deux langues et ne pose pas de réel problème de traduction: par exemple, paléoanthropologie et paleoantropologija.

Comme pour la partie phraséologique discursive, les termes (simples ou complexes) sont extraits de deux corpus comparables. Ces fiches sont ensuite saisies dans la base. La fiche complète (Tableau 7) comporte les éléments suivants: le terme en langue source; une indication sur la langue source; la catégorie grammaticale du terme; le(s) domaine(s); le(s) contexte(s); les sources $\mathrm{du} /$ des contextes; la définition (sa source); les collocations; les concurrents; les antonymes; les hyperonymes, méronymes, etc.; le statut du terme; la note technique; la note linguistique; la note de traduction; $1(\mathrm{es})$ équivalent(s). Le même schéma est saisi pour la langue cible. 
Tableau 7: Exemple de fiches terminologiques comparables

\begin{tabular}{|c|c|}
\hline Fiche $1-$ langue française & Fiche $2-$ langue slovène \\
\hline $\begin{array}{l}\text { LIGAMENT CROISE } \\
\text { nom, masculin, terme }\end{array}$ & $\begin{array}{l}\text { KRIZNA VEZ } \\
\text { nom, féminin, terme }\end{array}$ \\
\hline $\begin{array}{l}\text { Domaine(s): } \\
\text { — Sciences médicales. Médecine. Hygiène. } \\
\text { Pharmacie [CDU 2004] }\end{array}$ & $\begin{array}{l}\text { Domaine(s): } \\
\text { — Sciences médicales. Médecine. } \\
\text { Hygiène. Pharmacie [CDU 2004] }\end{array}$ \\
\hline $\begin{array}{l}\text { Auteur de la fiche: Katja Mavrič Bordon, } \\
\text { Étudiant(e) en Master de traduction, } \\
\text { 2017-2018 } \\
\text { Définition(s): } \\
\text { _ deux ligaments postérieurs du genou: le } \\
\text { ligament croisé antérieur et le ligament } \\
\text { croisé postérieur } \\
\text { [Source: ligaments croisés — Le grand dic- } \\
\text { tionnaire terminologique } \\
\text { (http://www.granddictionnaire.com/ficheO } \\
\text { qlf.aspx?Id_Fiche=8414667)] }\end{array}$ & $\begin{array}{l}\text { Auteur de la fiche: Katja Mavrič Bordon, } \\
\text { Étudiant(e) en Master de traduction, } \\
2017-2018 \\
\text { Définition(s): } \\
\text { _ } \\
\text { vezivo v obliki snopa ali ovojnice, ki } \\
\text { povezuje dve kosti ali več kosti, } \\
\text { hrustanec ali druge strukture } \\
\text { [Source: križna vez — Termania } \\
\text { (http://www.termania.net/slovarji/ } \\
\text { slovenski-medicinski-slovar/5524711/ } \\
\text { ligament)] }\end{array}$ \\
\hline $\begin{array}{l}\text { Contexte(s) / Expressions spécialisées } \\
\text { (collocations): } \\
\text { Le ligament croisé postérieur (LCP) est } \\
\text { situé au milieu du genou (il fait partie du } \\
\text { "pivot central"). Placé dans l'échancrure du } \\
\text { fémur, véritable cavité au milieu du genou. } \\
\text { [Source: Vincent Chassing — LCP } \\
\text { (http://genou.com/lcp/lcpdetaille.ht)] } \\
\text { La reconstruction chirurgicale du ligament } \\
\text { croisé antérieur, dont le but est de rétablir } \\
\text { la stabilité et la biomécanique du genou lésé, } \\
\text { est un sujet central en traumatologie du } \\
\text { sport. [Source: V. B. Duthon, G. Messerli, } \\
\text { J. Menetrey — Reconstruction du ligament } \\
\text { croisé antérieur: indications et techniques - } \\
\text { Revue Médicale Suisse - volume 4 } \\
\text { (https://www.revmed.ch/RMS/2008/RMS- } \\
\text { 184/Reconstruction-du-ligament-croise- } \\
\text { anterieur-indications-et-techniques) (2008)] } \\
\text { Le ligament croisé antérieur (LCA) est un } \\
\text { stabilisateur essentiel de l'articulation du } \\
\text { genou. Il empêche la translation antérieure } \\
\text { du tibia par rapport au fémur et agit comme } \\
\text { frein secondaire à sa rotation Rekonstrukcija } \\
\text { sprednje križne vezi — Artros } \\
\text { (https://artros.si/operativni_posegi/rekon } \\
\text { strukcija_sprednje_krizne_vezi/)] }\end{array}$ & $\begin{array}{l}\text { Contexte(s) / Expressions spécialisées } \\
\text { (collocations): } \\
\text { Zadnja križna vez je debelejša in dvakrat } \\
\text { močnejša od ACL. Predstavlja os za } \\
\text { zunanjo in notranjo rotacijo kolena. } \\
\text { [Source: POŠKODBA SPREDNJE } \\
\text { KRIŽNE VEZI KOLENA — GoFit } \\
\text { (https://www.gofit.si/poskodba- } \\
\text { sprednje-krizne-vezi-kolena/)] } \\
\text { Sprednja križna vez veže sprednjo } \\
\text { interkondilarno kotanjico golenice z } \\
\text { medialno ploskvijo lateralnega } \\
\text { kondila stegnenice. Ima izredno } \\
\text { pomembno stabilizacijsko vlogo, ker } \\
\text { preprečuje drsenje golenice navzpred, } \\
\text { preveliko zunanjo rotacijo in } \\
\text { hiperekstenzijo kolena. [Source: } \\
\text { POŠKODBA SPREDNJE KRIŽNE } \\
\text { VEZI KOLENA — GoFit } \\
\text { (https://www.gofit.si/poskodba- } \\
\text { sprednje-krizne-vezi-kolena/)] } \\
\text { Artroskopska rekonstrukcija križnih } \\
\text { vezi z avtolognim kitnim presadkom } \\
\text { je uveljavljena metoda izbora za } \\
\text { zdravljenje pretrganja sprednje križne } \\
\text { vezi. Pri posegu kirurg nadomesti } \\
\text { pretrgano sprednjo križno vez s kitnim } \\
\text { presadkom, običajno z avtologno kito } \\
\text { mišice semimembranosus in gracilis, ki }\end{array}$ \\
\hline
\end{tabular}




\begin{tabular}{|c|c|}
\hline & $\begin{array}{l}\text { jo odvzame med posegom iz zadnje } \\
\text { stegenske lože. [Source: Rekonstrukcija } \\
\text { sprednje križne vezi - Artros } \\
\text { (https://artros.si/operativni_posegi/re } \\
\text { konstrukcija_sprednje_krizne_vezi/)] }\end{array}$ \\
\hline $\begin{array}{c}\text { Traductions: } \\
\text { - (Français) ligament croisé nom, } \\
\text { masculin, terme }\end{array}$ & $\begin{array}{r}\text { Traductions: } \\
\text { - }\end{array}$ \\
féminin, terme
\end{tabular}

Il est également possible de ne fournir qu'une fiche glossaire qui porte sur les termes du texte. La fiche glossaire contient alors le terme en langue source; une indication sur la langue source; la catégorie grammaticale du terme; le domaine; la définition (sa source); les collocations; les concurrents; la note de traduction; 1(es) équivalent(s) et inversement pour la langue cible.

En final, l'étudiant remet un dossier à la fin du semestre qui est composé d'une introduction où il présente le domaine, justifie l'intérêt de son choix et la méthode de travail sur corpus. Dans la partie commentaire, il explique et développe sa démarche terminologique, aussi bien pour la traduction que pour la collecte des sources et l'élaboration des ressources terminologiques. Enfin, l'apprenant tire des conclusions sur son travail. D'autres activités pratiques peuvent également être proposées ou consignées dans le dossier. L'étudiant peut, par exemple, tenir un journal durant le semestre où il consigne en détail le déroulement de son travail, les problèmes qu'il a rencontrés, comment il les a résolus ou pas, ou encore ajouter des suggestions d'adaptation de la méthode employée au traitement de son domaine. Ces informations lui permettent de rédiger au fur et à mesure une analyse synthétique des particularités terminologiques du domaine choisi. Il peut également construire l'arborescence du domaine ou/et traduire une dizaine de lignes, voire plus, à partir d'un texte de son choix, etc. Par ailleurs, des présentations orales à l'aide de powerpoint peuvent permettre un échange en langue étrangère et un partage des connaissances acquises dans un domaine particulier.

\section{Synthèse des résultats}

\subsection{Fiches phraséologiques}

À l'issue du travail de création des fiches phraséologiques, nous notons que les étudiants ont saisi et traduit principalement des collocations génériques appartenant aux structures lexico-grammaticales suivantes:

(1) $\mathrm{Vb}+\mathrm{N}$ : les catégories grammaticales sont les mêmes dans les deux langues (sprejeti ukrepe/adopter les mesures).

(2) Adj+N qui correspond principalement à la structure grammaticale française N+Adj (pravična obravnave/le traitement équitable). Toutefois, certaines 
collocations de cette catégorie sont également traduites en français par la catégorie grammaticale $\mathrm{N}+$ prép (de) $+\mathrm{N}$ (tiskovna konferenca/conférence de presse).

(3) La troisième catégorie la plus fréquente concerne la construction grammaticale slovène $\mathrm{N}+\mathrm{N}$ qui est traduite par quatre catégories grammaticales différentes:

- N+prép $+\mathrm{N}$ (varstvo potrošnikov/protection du consommateur)

- N+adj (združiteo družine/regroupement familial)

- $\mathrm{Vb}+$ prép $+\mathrm{N}$ (odpoved imuniteti/renoncer à l'immunité)

- Construction nominale (sklenitev sporazuma/la mise en place de l'accord)

La première catégorie $(\mathrm{Vb}+\mathrm{N})$ est la plus fréquente, à raison de $51 \%$; la deuxième catégorie comprenant la structure française $\mathrm{Adj}+\mathrm{N}$ traduite par la structure slovène inversée équivaut à 19\% et les autres structures à 30\%.

\subsection{Fiches terminologiques}

Les dossiers remis par les étudiants nous permettent de recenser et de classer quelques différences. La médecine étant un domaine apprécié des étudiants, nous prenons l'exemple des termes médicaux français et slovènes. Les résultats ont été mis en commun et nous en présentons un extrait dans le Tableau 8:

Tableau 8: Récapitulatif des résultats des étudiants dans le domaine de la médecine

\begin{tabular}{|l|l|}
\hline Type & Exemples tirés des corpus des étudiants \\
\hline $\begin{array}{l}\text { 1. Termes savants presque identiques: } \\
\text { différences orthographiques (chute de } \\
\text { l'accent aigu ou grave) }\end{array}$ & \\
$\begin{array}{l}\text { 1a. même prononciation, la finale est une } \\
\text { consonne en slovène }\end{array}$ & $\begin{array}{l}\text { carcinome / karcinom } \\
\text { amyloïde / amiloid } \\
\text { auxine / avksin } \\
\text { papillomavirus / papilomavirus } \\
\text { viroïde / viroid } \\
\text { cystatine C / cistatin C } \\
\text { cytostatique / citostatik } \\
\text { allèle / alel } \\
\text { amphotère / amfoter } \\
\text { 1b. ajout du - a final en slovène } \\
\text { coccidiose / kokcidioza } \\
\text { myxomatose / miksomatoza } \\
\text { papillomatose / papilomatoza } \\
\text { agnosie / agnozija } \\
\text { cyanose / cianoza } \\
\text { épilepsie / epilepsija }\end{array}$ \\
\hline
\end{tabular}


1c. la terminaison-gie [3i] devient -gija [gija] en slovène; la constrictive prépalatale [3] passe à l'occlusive vélaire $[\mathrm{g}]$ en position initiale ou interne

1d. dénasalisation et terminaison en $-a$ pour le substantif, et en -čen pour l'adjectif slovène

1e. suffixes différents

$$
\text { -té }=\text {-ost }
$$

-isque $=$-iskus

-ite $=$-itis

-isme $=-\mathrm{ika}$

-tion et - sion $=-$ ija

2. Inversion grammaticale: $\mathrm{N}+\mathrm{adj} .=\mathrm{adj} .+\mathrm{N}$

3. Termes différents

3a. termes savants différents

3b. termes vernaculaires différents

3c. expressions françaises traduites par un ou plusieurs termes slovènes otorrhée / otoreja

néphrectomie / nefrektomija

céphalométrie / cefalometrija

névroglie / nevroglija

génome / genom

diplégie / diplegija

éthologie / etologija

démence / demenca

endosymbiose / endosimbioza

nevrolinguistique / nevrolingvističen

eusocialité / evsocialnost

ménisque / meniskus

arthrite / artritis

eugénisme / evgenika

abscission / abscizija

rémission / remisija

démyélinisation / demielinizacija

malocclusion / malokluzija

ictère nucléaire / nuklearni ikterus

cortex préfrontal / prefrontalni korteks

neurosciences cognitives / kognitivna

nevroznanost

axone / nevrit

chimiothérapie / kemoterapija

thyroïde / ščitnica

hémorragie / krvavitev

homolatéral / ipsilateralen

surrénale / nadledvičnica

gale / garje

rechute / relaps

nutriment / hranilo

prématurité / nedonošenost

déjection / blato

appareil urinaire / sečila

enzyme de restriction / restriktaza

ligament croisé / križna vez

plaque sénile / senilna leha

infirmité motrice cérébrale/ cerebralna paraliza

dégénérescence neurofibrillaire /

nevrofibrilarna pentlja

surexpression du gène / čezmerno izražanje

gena

noyaux gris centraux / bazalni gangliji v

osrednjem delu možganov

substance blanche / bela možganovina 
Nous notons que les étudiants n'ont saisi aucun terme parfaitement identique dans les deux langues. Le Tableau 8 indique que les termes savants sont les plus fréquents dans les deux langues, même si en français la différence est plus significative (90\%) qu'en slovène $(80 \%)$. La majorité des termes savants prennent une orthographe différente tandis que d'autres diffèrent de par leur traduction. La plupart des termes et des collocations sont traduits par un emprunt savant qui rappelle parfois le mot d'origine latine ou grecque: par exemple, le terme ictère vient du latin icterus ou du grec ikteros, ce qui donne en slovène ikterus. On en conclut que le slovène médical semble emprunter directement au latin ou au grec.

\section{Conclusion}

La création du projet lexicographique, la compilation de données, l'activité de traduction, l'approche contrastive, etc. se référant à la paire de langues françaisslovène est une démarche innovante en Slovénie. L'originalité du dictionnaire ARTES réside dans son approche globale qui réunit des éléments linguistiques, terminologiques, phraséologiques et discursifs pertinents pour la traduction et la communication en langue de spécialité. La base de données offre un cadre intéressant pour une approche bilingue ou multilingue. Elle permet en outre de mener des recherches sur une variété de phénomènes linguistiques. Dans le cas présent et d'une part, elle nous a permis de développer une nouvelle approche pédagogique en lien avec le futur contexte professionnel de l'étudiant. En deux ans, cette expérience lexicographique a permis de créer dans la base ARTES plus de 200 fiches terminologiques et plus de 400 fiches phraséologiques françaisslovènes. D'autre part, cela permet de mener des recherches à partir des données terminologiques et phraséologiques qui sont stockées par les apprenants dans la base. La base peut ainsi être utilisée comme modèle pour l'analyse de la terminologie et de la phraséologie discursive pour la paire français-slovène dans le domaine de la traduction spécialisée.

Cette approche lexicographique s'est avérée être une étape à la fois formatrice et pertinente du point de vue de la traduction et de la communication spécialisée. L'étudiant met en pratique une méthode de traitement lexicographique, développée par une université française, ce qui permet par ailleurs d'approfondir à plus ou moins long terme les liens inter-universitaires. Il apprend à développer une réflexion méthodologique, mais il pourra aussi être en mesure d'adapter si nécessaire les méthodes et les outils à ses propres besoins. Enfin, les dossiers remis par l'étudiant mettent en évidence la progression de la réflexion terminologique et phraséologique, mais aussi sa maîtrise de la langue française et ses compétences en traduction. Cela permet de mettre en évidence les points forts des dossiers, de tirer des conclusions lexicographiques sur un ou plusieurs domaines spécialisés, mais aussi de souligner le bilan individuel et la progression de l'étudiant dans son apprentissage d'une 
langue étrangère et d'une méthode de traduction qui diffère de la méthode classique.

\section{Remerciements}

La compilation de ressources lexicographiques français-slovène dans la base de données ARTES a bénéficié du soutien du Département de linguistique appliquée et d'études interculturelles - UFR EILA — de l'Université Paris Diderot. Nous tenons à remercier l'équipe de recherche du département EILA, notamment Mojca Pecman, Nicolas Froeliger, Natalie Kübler, Geneviève Bordet, Alexandra Volanschi et Brice Bricaud pour la mise à disposition de la base de données, leurs concours précieux et leur participation active au projet.

\section{Références bibliographiques}

Baker, M. 1993. Corpus Linguistics and Translation Studies. Implications and Applications. Baker, M., G. Francis and E. Tognini-Bonelli (Éds.). 1993. Text and Technology: In honour of John Sinclair: 233-250. Philadelphia: John Benjamins

Boers, F., M. Demecheleer et J. Eyckmans. 2004. Etymological Elaboration as a Strategy for Learning Idioms. Bogaards, P. and B. Laufer (Éds.). 2004. Vocabulary in a Second Language: Selection, Acquisition and Testing: 53-78. Amsterdam: John Benjamins.

Bowker, L. et J. Pearson. 2002. Working with Specialized Language: A Practical Guide to Using Corpora. London: Routledge.

Cavalla, C. 2009. La phraséologie en classe de FLE. Les Langues Modernes. Association des professeurs de langues vivantes (APLV), pp. en ligne. https://hal.archives-ouvertes.fr/hal-00699916.

Detry, F. 2008. Pourquoi les murs auraient-ils des oreilles? Vers un apprentissage par l'image des expressions idiomatiques en langue étrangère. Synergies Espagne 1: 205-218.

Durán-Muñoz, I. 2010. Specialized Lexicographical Resources: A Survey of Translators' Needs. Granger, S. and M. Paquot (Éds.). 2010. eLexicography in the 21st Century: New Challenges, New Applications. Proceedings of ELEX2009: 55-66. Louvain-la-Neuve: Presses universitaires de Louvain.

Galisson, R. 1983. Des mots pour communiquer: éléments de lexicométhodologie. Paris: CLE International.

Galisson, R. 1991. De la langue à la culture par les mots. Paris: CLE.

Gantar, P. 2015. Leksikografski opis slovenščine v digitalnem okolju. [Dictionary of Modern Slovene: Problems and Solutions.] Zbirka Sporazumevanje. Ljubljana: Ljubljana University Press, Faculty of Arts.

Gorjanc, V., P. Gantar, I. Kosem et S. Krek (Éds.). 2017. Dictionary of Modern Slovene: Problems and Solutions. Prevodoslovje in uporabno jezikoslovje. Ljubljana: Ljubljana University Press, Faculty of Arts.

Grad, A. (1971) 2004. Francosko-slovenski slovar. Ljubljana: Cankarjeva založba.

Jakobsen, A.L. 1999. Logging Target Text Production with Translog. Hansen, G. (Éd.). 1999. Probing the Process in Translation: Methods and Results): 9-20. Copenhagen Studies in Language 24. Copenhagen: Samfundslitteratur. 
Jakobsen, A.L. 2000. Understanding the Process of Translation: The Contribution of Time-Delay Studies. Englund Dimitrova, B. (Éd.). 2000. Översättning och tolkning: 155-172. Stockholm: ASLA.

Jesenik, V. et N. Dembskij. (1990) 2008. Slovensko-francoski slovar. Ljubljana: Državna založba Slovenije.

Kübler, N. 2003. Corpora and LSP Translation. Zanettin, F., S. Bernardini and D. Stewart (Éds.). 2003. Corpora in Translator Education: 25-42. Manchester: St Jerome.

Kübler, N. 2011. Corpora, Language, Teaching, and Resources: From Theory to Practice. Bern: Peter Lang.

Kübler, N. et M. Pecman. 2012. The ARTES Bilingual LSP Dictionary: From Collocation to Higher Order Phraseology. Granger, S. and M. Paquot (Éds.). 2012. Electronic Lexicography: 187-209. Oxford: Oxford University Press.

Ledinek, N, K. Ahačič et A. Perdih. 2015. Fran: slovarji Inštituta za slovenski jezik Frana Ramovša ZRC $S A Z U$, Vodnik. [A Guide to Fran: The Dictionaries from the Fran Ramovš Institute of the Slovenian Language in the Research Center of the Slovenian Academy of Sciences and Arts.] Zbirka Fran. Ljubljana: Založba ZRC.

L'Homme, M.-C. 2004. La terminologie: principes et techniques. Montréal: Les Presses de l'Université de Montréal.

Mavrič Bordon, K. 2019. Uporabni elektronski viri za prevajanje terminologije s področja krasoslovja: magistrsko delo. Ljubljana: Filozofska fakulteta.

Meyer, I. et K. Mackintosh. 1996. The Corpus from a Terminographer's Viewpoint. International Journal of Corpus Linguistics 1(2): 257-285.

Morin, E. et B. Daille. 2006. Comparabilité de corpus et fouille terminologique multilingue. Traitement Automatique des Langues. ATALA 47(1): 113-136.

Morin, E. et B. Daille. 2012. Compositionnalité et contextes issus de corpus comparables pour la traduction terminologique. TALN 2: 141-154.

Olohan, M. 2004. Introducing Corpora in Translation Studies. London/New York: Routledge.

Pavičić Takač, V. 2008. Vocabulary Learning Strategies and Foreign Language Acquisition. Second Language Acquisition 27. Clevedon/Buffalo/Toronto: Multilingual Matters.

Pecman, M. 2007. Phraseology and Didactics: Lexical Combinatorics as a Teaching Tool of English for French Students in Scientific Disciplines. Kržišnik, E. and W. Eismann. 2007. Frazeologija v Jezikoslovju in Drugih Vedah/Phraseologie in der Sprachwissenschaft und anderen Disziplinen/ Phraseology in Linguistics and Other Branches of Science: 349-363. Strunjan: European Society of Phraseology (EUROPHRAS).

Pecman, M. 2012. Etude lexicographique et discursive des collocations en vue de leur intégration dans une base de données terminologiques. Terminology, Phraseology and Translation. Special Issue of The Journal of Specialized Translation (JoSTrans) 18: 113-138.

Pecman, M. et C. Gledhill. 2018. How Trainee Translators and Their Teachers Deal with Phraseological Units in the ARTES Database. Equivalences. 45(1-2): 235-257.

Pecman, M. et N. Kübler. 2011. ARTES: An Online Lexical Database for Research and Teaching in Specialized Translation and Communication. Proceedings of the First International Workshop on Lexical Resources (WoLeR) 2011 at ESSLLI, August 1-5, 2011: 87-93. Ljubljana: Ljubljana University Press.

Perko, G. 2006. Francosko-slovenski, slovensko-francoski žepni slovar. Ljubljana: Cankarjeva založba. 
Perko, G. 2007. Slovensko-francoski evropski slovar. Ljubljana: Cankarjeva založba.

Simonnet, D. et A. Tutin. 2019. Utiliser la linguistique de corpus pour renforcer les compétences des étudiants dans l'analyse syntaxique. Langue française 203: 83-99.

Snoj, J. 2004. Tipologija slovarske večpomenskosti slovenskih samostalnikov. [The Lexicographic Treatment of Polysemous Nouns in Slovenian.] Zbirka Linguistica et philologica. Ljubljana: Založba ZRC SAZU.

Srebnik, A. 2015. Jezikovnotehnološki postopek obračanja dvojezičnih slovarjev. [The Technology and Linguistics behind the Process of Reversing Bilingual Dictionaries.] Praha: Verbum.

Szczepaniak, R. et R. Lew. 2011. The Role of Imagery in Dictionaries of Idioms. Applied Linguistics 32(3): 323-347.

Tréville, M.-C. 2000. Vocabulaire et apprentissage d 'une langue seconde: recherches et théories. Montréal, Québec: Éditions Logiques.

Udovič, B. 2018. Zgodovina (gospodarske) diplomacije. Ljubljana: Založba FDV.

Volanschi, A. 2007. Outils informatiques et ressources électroniques pour les traducteurs. Tribune internationale des langues vivantes (TILV) 43(2): 24-39.

Vrbinc, A., D.M.T.Cr. Farina et M. Vrbinc. 2018. Objectivity, Prescription, Harmlessness, and Drudgery: Reflections of Lexicographers in Slovenia. Lexikos 28: 361-383.

Žagar Karer, M. 2011. Terminologija med slovarjem in besedilom: analiza elektrotehniške terminologije. [Terminology from the Text to the Dictionary: Analysis of Electro-technical Terminology.] Zbirka Linguistica et philologica 26. Ljubljana: Založba ZRC. 